\title{
18F-Fluorodeoxyglucose Positron Emission Tomography Is Useful in the Evaluation of Prognosis in Retroperitoneal Sarcoma
}

\author{
Toru Wakamatsu ${ }^{1,2}$, , Yoshinori Imura ${ }^{1,2}$, Hironari Tamiya ${ }^{1,2}$, Toshinari Yagi ${ }^{2}$, Naohiro Yasuda ${ }^{1}$, Sho Nakai ${ }^{1,2}$, \\ Takaaki Nakai $^{3}$, Hidetatsu Outani ${ }^{1}{ }^{10}$, Kenichiro Hamada ${ }^{1}$, Shigeki Kakunaga ${ }^{3}$, Nobuhito Araki ${ }^{2}$, \\ Takafumi Ueda ${ }^{3}$ and Satoshi Takenaka ${ }^{1,2}$
}

1 Department of Orthopaedic Surgery, Osaka University Graduate School of Medicine, 2-2 Yamadaoka Suita, Osaka 565-0871, Japan; y.imura@mc.pref.osaka.jp (Y.I.); hironari.tamiya@oici.jp (H.T.); n.yasuda@ort.med.osaka-u.ac.jp (N.Y.); nakai-sho@mc.pref.osaka.jp (S.N.); h-otani@ort.med.osaka-u.ac.jp (H.O.); hamada-ke@mc.pref.osaka.jp (K.H.); takenaka-sa@mc.pref.osaka.jp (S.T.)

2 Department of Musculoskeletal Oncology Service, Osaka International Cancer Institute, 3-1-69 Otemae, Osaka 541-8567, Japan; yagi-to@mc.pref.osaka.jp (T.Y.); n.araki@ashiya-hosp.com (N.A.)

3 Department of Orthopaedic Surgery, National Hospital Organization Osaka National Hospital, 2-1-14 Hoenzaka, Osaka 540-0006, Japan; nakai.takaaki.mz@mail.hosp.go.jp (T.N.); kakunaga.shigeki.ya@mail.hosp.go.jp (S.K.); s.uedat@soreiyu.net (T.U.)

* Correspondence: wakamatu-to@mc.pref.osaka.jp

\section{check for} updates

Citation: Wakamatsu, T.; Imura, Y.; Tamiya, H.; Yagi, T.; Yasuda, N.; Nakai, S.; Nakai, T.; Outani, H.; Hamada, K.; Kakunaga, S.; et al. 18F-Fluorodeoxyglucose Positron Emission Tomography Is Useful in the Evaluation of Prognosis in Retroperitoneal Sarcoma. Cancers 2021, 13, 4611. https://doi.org/ $10.3390 /$ cancers 13184611

Academic Editor: Armita Bahrami

Received: 10 August 2021

Accepted: 10 September 2021

Published: 14 September 2021

Publisher's Note: MDPI stays neutral with regard to jurisdictional claims in published maps and institutional affiliations.

Copyright: (c) 2021 by the authors. Licensee MDPI, Basel, Switzerland. This article is an open access article distributed under the terms and conditions of the Creative Commons Attribution (CC BY) license (https:/ / creativecommons.org/licenses/by/ $4.0 /)$.
Simple Summary: Retroperitoneal sarcomas are difficult malignancies to treat because complete surgical resection is the only effective treatment option, but it is difficult to secure sufficient surgical margins. It is essential for developing a treatment strategy to assess tumor aggressiveness and predict prognosis for patients. However, the aggressiveness of retroperitoneal sarcomas before treatment cannot be fully evaluated. In patients with resectable soft tissue sarcomas or several carcinomas, SUV evaluated with FDG-PET has been reported to be a valuable prognostic parameter. However, the correlation between SUVmax on FDG-PET and the prognosis of several histological subtypes in retroperitoneal sarcoma, including dedifferentiated liposarcoma, well-differentiated liposarcoma, and leiomyosarcoma, remains uncertain. This study revealed that SUVmax calculated with FDG-PET was useful as a prognostic factor in retroperitoneal sarcoma, especially in dedifferentiated liposarcoma and Grade2 retroperitoneal sarcoma.

Abstract: Background: Retroperitoneal sarcomas are rare neoplasms that occur in the retroperitoneum. Complete surgical resection is the only effective treatment option. The prediction of prognosis by histological diagnosis has not yet been established. The purpose of this study was to identify the usefulness of [18-F] fluorodeoxyglucose (FDG) positron emission tomography (PET) imaging for validating the prognosis of retroperitoneal sarcoma (RPS) established by histological diagnosis. Methods: We retrospectively reviewed 201 patients with RPS treated at the Osaka International Cancer Institute between 2010 and 2021. We extracted the clinical data, including standardized uptake values (SUVs), evaluated with FDG-PET, and statistically analyzed the data. Results: The median age of patients was 64 years (range, 31-85 years). A total of 101 (50.2\%) patients were men, and $100(49.8 \%)$ were women. Surgical resection was performed in $155(77.1 \%)$ patients. On histological analysis, $75(37.3 \%), 52(25.9 \%)$, and 29 (14.4\%) patients were diagnosed with dedifferentiated liposarcoma, well-differentiated liposarcoma, and leiomyosarcoma, respectively. The median survival time for patients with high maximum SUV (SUVmax) $(\geq 4)$ or low SUVmax $(<4)$ was 275.8 months and 79.5 months, respectively. Furthermore, among the patients with dedifferentiated liposarcoma, the overall survival rate for patients with high SUVmax $(\geq 4)$ was significantly lower than that of those with low SUVmax $(<4)$. Conclusions: The present study demonstrated that SUVmax calculated with FDG-PET was useful as a prognostic factor in RPS, especially in dedifferentiated liposarcoma and Grade2 RPS. To devise a treatment strategy for RPS, SUVmax during FDG-PET scan may be considered for clinical assessment. 
Keywords: retroperitoneal sarcoma; FDG-PET; SUVmax; dedifferentiated liposarcoma; leiomyosarcoma

\section{Introduction}

Retroperitoneal sarcoma (RPS) is a rare mesenchymal tumor consisting of approximately $10 \%$ of all soft tissue sarcomas (STS) [1-3]. There are many histological subtypes of RPS; approximately $75-80 \%$ of the tumors are diagnosed as dedifferentiated liposarcoma (DDLPS), well-differentiated liposarcoma (WDLPS), or leiomyosarcoma (LMS) [4,5]. Although complete resection by surgery is the only effective treatment to improve prognosis, it is difficult to secure sufficient surgical margins because of the tumor size and location of the RPS [6,7]. In contrast, chemotherapy and radiotherapy do not play a pivotal role in the treatment strategy for RPS [8,9]. Recently, a randomized clinical trial (EORTC-62092) failed to demonstrate the benefit from preoperative radiotherapy for RPS, except for the liposarcoma subgroup [9]. Thus far, unlike soft tissue sarcoma of the extremities, multimodality treatment of surgical resection combined with chemotherapy and/or radiotherapy for RPS has not been established. The most beneficial treatment strategy for RPS might be repeated excision for primary and recurrent or metastatic tumors, and chemotherapy and/or radiotherapy are considered effective when the tumors become unresectable [6].

To develop a treatment strategy and predict prognosis for patients with malignant tumors, assessment of tumor aggressiveness is essential, determined by pathological diagnosis and Grade. Furthermore, the clinical tumor stage and size are evaluated, and then the treatment strategy is designed. However, the aggressiveness of RPS before treatment cannot be fully assessed because a biopsy is often not even performed. However, if a biopsy was performed, the biopsy tissue sometimes does not reflect the tumor aggressiveness because of the tumor size and heterogeneity of RPS. Various histological subtypes of RPS are often treated similarly without appropriate clinical assessment.

Positron emission tomography (PET) using 2-fluoro-2-deoxy-D-glucose (FDG) is an imaging tool clinically used for early detection of primary and metastatic tumors with high sensitivity by evaluating tumor glucose metabolism [10,11]. Standardized uptake values (SUVs) reflecting FDG uptake by tumors can be calculated. In patients with resectable STS, SUV has been reported to be a valuable prognostic parameter similar to several carcinomas, including lung, colon, and pancreas [12,13]. However, the utility of PET for predicting the prognosis of RPS is not clearly understood. A study reported that histologic subtype and Federation Nationale des Centres de Lutte le Cancer (FNCLCC) Grade in patients with retroperitoneal liposarcoma could be assessed by the maximum standardized uptake value (SUVmax) measured on FDG-PET [14]. Although another study showed that SUVmax on FDG-PET improved outcome prediction in retroperitoneal liposarcoma, it did not reveal that SUVmax was a valuable prognostic factor because all histological subtypes of liposarcomas in RPS were included and equally analyzed with the same stats [15]. WDLPS is known to have low malignant potential, but DDLPS is a high-grade malignancy. To date, the correlation between SUVmax on FDG-PET and the prognosis of DDLPS in RPS have not been clearly demonstrated. Likewise, the correlation between SUVmax on FDG-PET and the prognosis of other histological subtypes in RPS remains uncertain.

This study proposed that FDG-PET is a good tool for evaluating the early detection of tumors and tumor aggressiveness in RPS. We aimed to clarify that SUVmax measured with FDG-PET predicted the aggressiveness and prognosis of DDLPS and other histological subtypes in RPS. We also investigated the involvement of SUVmax in the clinical outcome of RPS patients compared with other candidate prognostic factors.

\section{Materials and Methods}

Retroperitoneal tumors were detected in 252 patients between 2010 and 2021 at the Osaka International Cancer Institute. We excluded 51 patients in this retrospective study who were lost to follow-up without treatment or diagnosed with benign tumors, including 
lipoma or schwannoma. As a result, 201 patients with RPS were recruited and analyzed in the current study. We also included patients who were clinically diagnosed with retroperitoneal malignant tumors without biopsy for pathological diagnosis. We retrospectively reviewed their medical records. The data of 201 patients with RPS, including their clinicopathological characteristics (age, gender, metastasis at the first visit, pathological histology, tumor grade, tumor size, and SUVmax with FDG-PET), treatment modalities (surgical resection, surgical margin, chemotherapy, and radiotherapy), and clinical outcomes were investigated (Table 1). Musculoskeletal pathologists diagnosed almost all cases of RPS at our institute. FDG-PET was performed for 145 patients at our institute or referral hospitals. We extracted the data with the highest SUVmax, and, in almost all cases, there was no significant difference in the SUVmax for each patient during their observation periods.

Table 1. Clinicopathologic and treatment characteristics.

\begin{tabular}{|c|c|c|}
\hline Characteristic & & No. (\%) \\
\hline \multirow[t]{2}{*}{ Age, years } & Range & $31-85$ \\
\hline & Median & 64 \\
\hline \multirow[t]{2}{*}{ Gender } & Male & $101(50.2)$ \\
\hline & Female & $100(49.8)$ \\
\hline \multirow[t]{2}{*}{ Metastasis at the first visit } & Yes & $13(6.5)$ \\
\hline & No & $188(93.5)$ \\
\hline \multirow[t]{4}{*}{ Histology } & Dedifferentiated liposarcoma & $75(37.3)$ \\
\hline & Well-differentiated liposarcoma & $52(25.9)$ \\
\hline & Leiomyosarcoma & $29(14.4)$ \\
\hline & Other & $45(22.4)$ \\
\hline \multirow[t]{4}{*}{ Tumor grade } & Grade1 & $67(33.3)$ \\
\hline & Grade2 & $90(44.8)$ \\
\hline & Grade3 & $34(16.9)$ \\
\hline & NE & $10(5.0)$ \\
\hline \multirow[t]{4}{*}{ Tumor size } & $<5 \mathrm{~cm}$ & $37(18.4)$ \\
\hline & $\geq 5 \mathrm{~cm}$ and $<15 \mathrm{~cm}$ & $90(44.8)$ \\
\hline & $\geq 15 \mathrm{~cm}$ & $66(32.8)$ \\
\hline & $\mathrm{NE}$ & $8(4.0)$ \\
\hline \multirow[t]{5}{*}{ Surgical resection } & Yes & $155(77.1)$ \\
\hline & $\mathrm{R} 0$ and $\mathrm{R} 1$ & $80(51.6)$ \\
\hline & $\mathrm{R} 2$ & $40(25.8)$ \\
\hline & Unknown & $35(22.6)$ \\
\hline & No & $46(22.9)$ \\
\hline \multirow[t]{2}{*}{ Chemotherapy } & Yes & $85(42.3)$ \\
\hline & No & $116(57.7)$ \\
\hline \multirow[t]{2}{*}{ Radiotherapy } & Yes & $56(27.9)$ \\
\hline & No & $145(72.1)$ \\
\hline \multirow[t]{2}{*}{ SUVmax } & Range & $0-25.5$ \\
\hline & Median & 4 \\
\hline NE; not estimated & & \\
\hline
\end{tabular}

The median age of the patients was 64 years (range, 31-85 years). The analyzed patients consisted of 101 men and 100 women. The median follow-up period was 41.8 months (range, 0.8-293.1 months). Distant metastasis was observed in 13 patients with RPS at the first visit to the hospital. The histological diagnoses were as follows: 75 patients with dedifferentiated liposarcoma (DDLPS), 52 with well-differentiated liposarcoma (WDLPS), 29 with leiomyosarcoma (LMS), and 45 with other histological types of sarcoma, including undifferentiated pleomorphic sarcoma, myxoid liposarcoma, myxofibrosarcoma, and solitary fibrous tumor. For tumor grade, 67, 90, and 34 patients were diagnosed with Grade1, Grade2, and Grade3 tumors, respectively. The tumor size was less than $5 \mathrm{~cm}$ in 37 patients, between $5 \mathrm{~cm}$ and $15 \mathrm{~cm}$ in 90 patients, and $15 \mathrm{~cm}$ or more in 66 patients. Surgical resection was performed in 155 patients with R0-1 or R2 resection in 80 or 40 patients, respectively. Thirty-five patients' surgical margins were unknown; chemotherapy as neoadjuvant, adju- 
vant, or advanced stage therapy was administered to 85 patients, and radiotherapy was administered to 56 patients. The median SUVmax was 4 (range, 0-25.5). A summary of these clinicopathological and treatment characteristics is shown in Table 1.

We compared the overall survival rate (OS) or disease-free survival rate (DFS) of each group using the Kaplan-Meier method and log-rank test for statistical estimates in univariate analysis. OS was defined as the time from the initial presentation to death associated with the disease or the last follow-up. DFS was defined as the time from the first surgical resection to the first detection of local recurrence, distant metastasis, or the last follow-up. We excluded patients with distant metastasis at the initial referral or without definitive surgery from the DFS analysis. Multivariate analysis was performed using the Cox proportional hazard method with variables. Statistical significance was set at $p<0.05$. Statistical analyses were performed using EZR software (Saitama Medical Center, Jichi Medical University, Saitama, Japan), a graphical user interface for R (The R Foundation for Statistical Computing, Vienna, Austria). Ethical approval for this study was obtained from the institutional review board of the Osaka International Cancer Institute.

\section{Results}

The 5-year and 10-year OS rates of all 201 patients with RPS were $74.7 \%$ and $59.8 \%$, respectively. According to the histological subtypes, the 5-year OS rates were $94.5 \%, 70.8 \%$, $67.8 \%$, and $59.7 \%$, and the 10 -year OS was $90.6 \%, 48.6 \%, 44.5 \%$, and $45.5 \%$ for WDLPS, DDLPS, LMS, and other, respectively (Figure 1). The prognosis of patients with DDLPS, LMS, and other subtypes was similarly worse than those with WDLPS. On the other hand, the 5-year DFS rates were $35.9 \%, 19.9 \%, 20.5 \%$, and $42.2 \%$ for WDLPS, DDLPS, LMS, and others, respectively. These results were considered to reflect a high local recurrence rate in DDLPS and frequent distant metastasis in LMS compared to WDLPS and others.

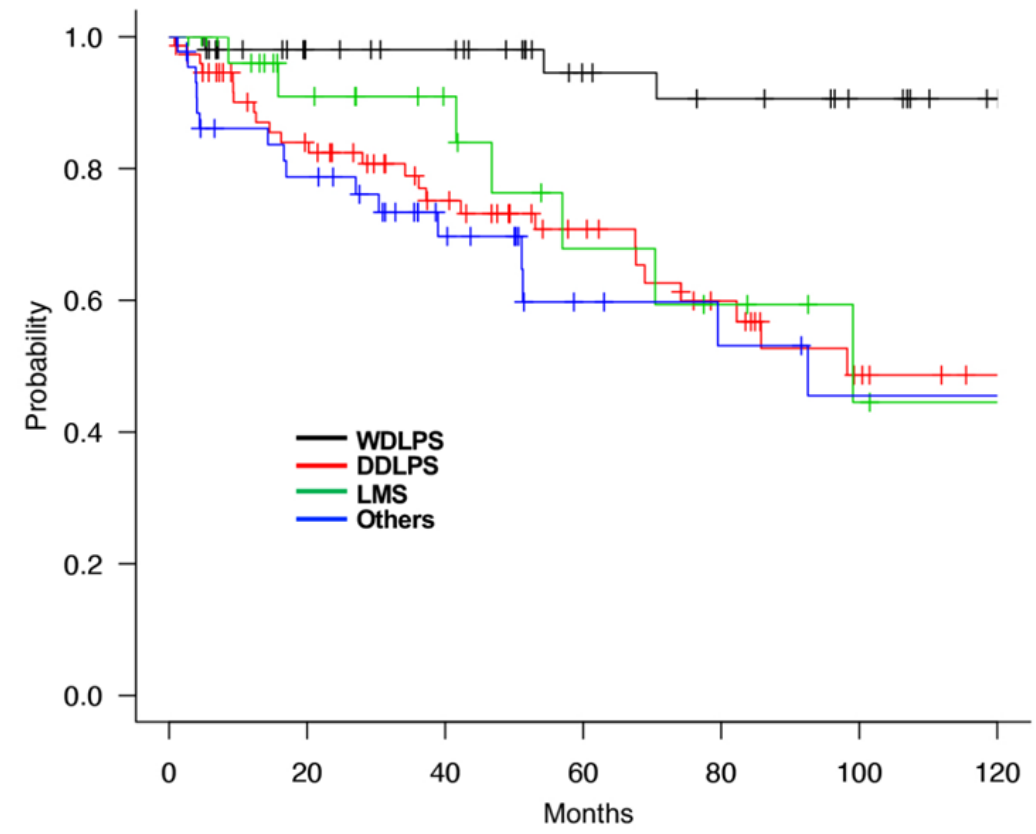

Figure 1. Overall survival (OS) of RPS patients by histological subtypes. WDLPS; well-differentiated liposarcoma, DDLPS; dedifferentiated liposarcoma, LMS; leiomyosarcoma.

We used the SUVmax threshold of 4 because this threshold was the median score of all patients with RPS in this study, and the prognosis of each group was the most significant with this setting. The 5-year OS of the SUVmax low $(<4)$ and SUVmax high $(\geq 4)$ groups were $92.9 \%$ and $58.4 \%$, respectively, showing that the SUVmax was a strong prognostic factor for RPS ( $p<0.001$, Figure 2a). Furthermore, the 5-year DFS rates of the SUVmax low and SUVmax high groups were $34.5 \%$ and $12.7 \%$, respectively $(p=0.027$, Figure $2 b)$. In 
addition, we compared the characteristics of the patients in the low- and high SUVmax groups. Most of the patients with Grade1 tumors had low SUVmax $(83.3 \%$, 35 out of 42$)$, and most of the patients with Grade3 tumors had high SUVmax (92.6\%, 25 out of 27). However, patients with Grade2 tumors were recruited in both SUVmax low and SUVmax high groups (Table 2). After that, we compared patient prognosis in the SUVmax low and SUVmax high groups by stratification with tumor Grade. It showed that patients with high SUVmax and Grade2 tumors had poor prognosis compared to those with low SUVmax and Grade2 tumors, and patients with high SUVmax and Grade3 tumors showed the poorest prognosis. There were insufficient numbers of patients with low SUVmax and Grade3 tumors or high SUVmax and Grade1 tumors for the abovementioned comparisons. (Figure 2c). These results suggested that SUVmax was a strong prognostic factor of at least Grade2 tumors in RPS.

a

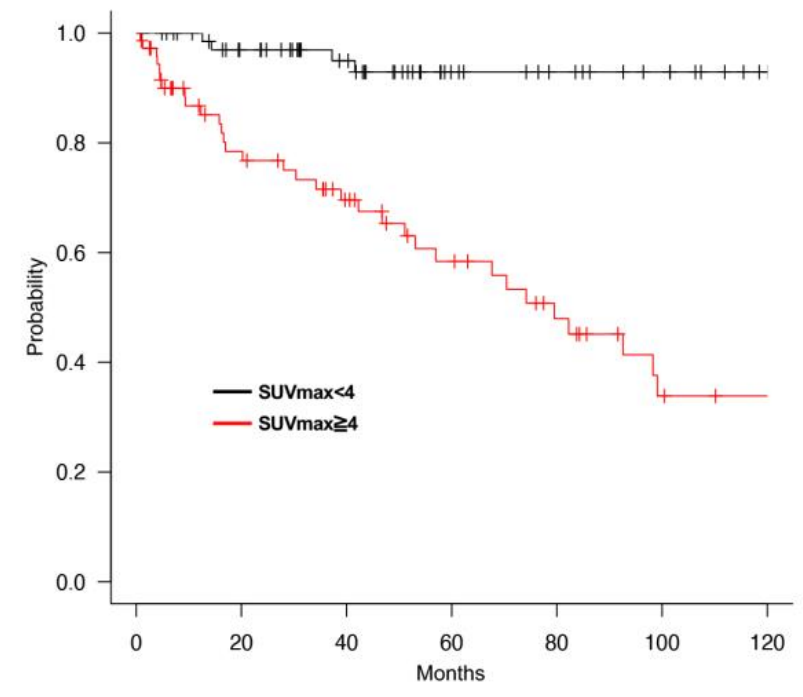

C

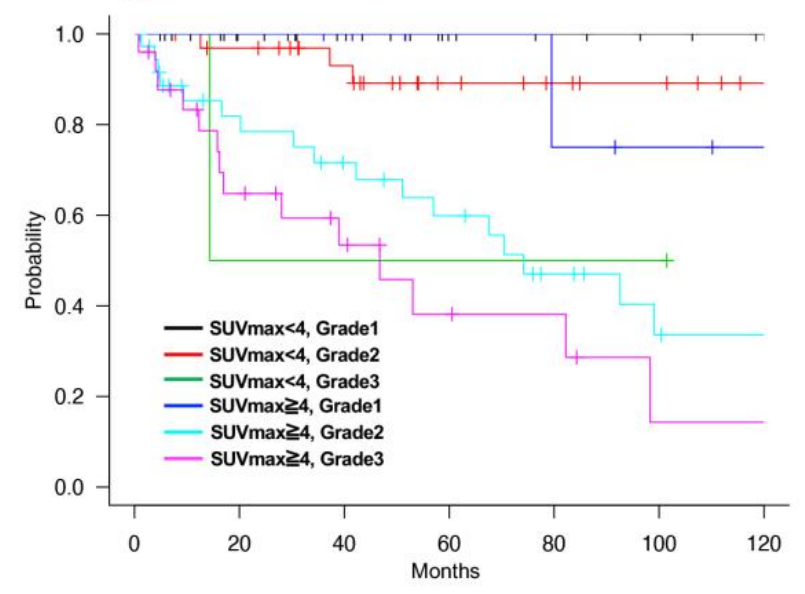

b

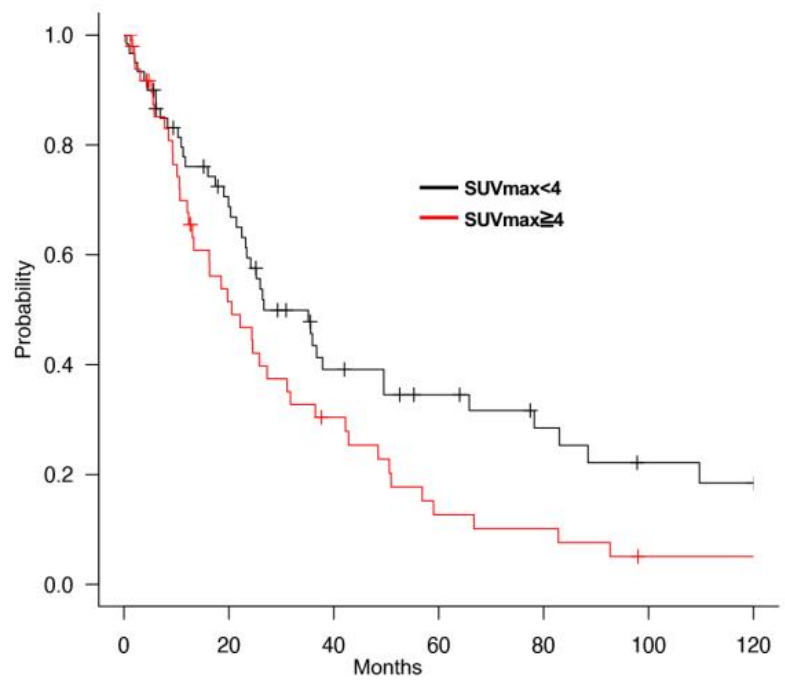

Figure 2. (a) OS of all RPS patients with SUVmax low $(<4)$ or SUVmax high $(\geq 4)$. (b) DFS of all RPS patients with SUVmax low $(<4)$ or SUVmax high $(\geq 4)$. (c) OS of RPS patients with SUVmax low and SUVmax high group by stratification with tumor grade.

We also performed analyses of OS with other variables using univariate analysis, which showed that gender, metastasis as the first visit, tumor Grade, histology, tumor size, surgical resection, chemotherapy, and radiotherapy were significant prognostic factors in RPS (Table 3). Similar to previous studies, patients who received chemotherapies or radiotherapies had a worse prognosis than those who did not receive these treatments, suggesting that the benefit of chemotherapy or radiotherapy was small for RPS (Table 3) [8,9]. 
Multivariate analyses demonstrated that a high SUVmax (hazard ratio $[\mathrm{HR}]=9.871 ; 95 \%$ confidence interval [CI]: 2.855-34.13; $p<0.001$ ) was independently correlated with shorter OS (Table 4). Surgical resection (hazard ratio [HR] $=0.165$; CI: 0.06428-0.4237; $p<0.001$ ) was also an independent prognostic factor for RPS (Table 4). Tumor Grade (hazard ratio $[\mathrm{HR}]=2.49 ; 95 \%$ CI: 0.505-12.28; $p=0.2624)$ was not an independent prognostic factor Meanwhile, in multivariate analyses, SUVmax was not independently correlated with DFS.

Table 2. Clinicopathologic and treatment characteristics between SUVmax low and SUVmax high.

\begin{tabular}{|c|c|c|c|}
\hline Characteristic & SUVmax Low $(n=72)$ & SUVmax High $(n=73)$ & $p$ Value \\
\hline Age, years (Range, median) & $34-83,63$ & $31-85,63$ & 0.814 \\
\hline Gender (male/female) & $26 / 46$ & $45 / 28$ & 0.002 \\
\hline Localized/metastatic & $70 / 2$ & $63 / 10$ & 0.017 \\
\hline Histology & & & $<0.001$ \\
\hline Well-differentiated liposarcoma & 29 & 4 & \\
\hline Dedifferentiated liposarcoma & 22 & 32 & \\
\hline Leiomyosarcoma & 7 & 17 & \\
\hline Other & 14 & 19 & \\
\hline Tumor grade & & & $<0.001$ \\
\hline Grade1 & 35 & 7 & \\
\hline Grade2 & 33 & 37 & \\
\hline Grade3 & 2 & 25 & \\
\hline NE & 2 & 4 & \\
\hline Tumor size & & & 0.961 \\
\hline$<5 \mathrm{~cm}$ & 17 & 13 & \\
\hline$\geq 5 \mathrm{~cm}$ & 54 & 59 & \\
\hline $\mathrm{NE}$ & 1 & 1 & \\
\hline Surgical resection (Yes/No) & $60 / 12$ & $51 / 22$ & 0.132 \\
\hline Chemotherapy (Yes/No) & $25 / 47$ & $45 / 28$ & 0.007 \\
\hline Radiotherapy (Yes/No) & $59 / 13$ & $31 / 42$ & 0.03 \\
\hline NE; not estimated & & & \\
\hline
\end{tabular}

Table 3. Analyses on survival in all RPS patients.

\begin{tabular}{|c|c|c|c|c|c|}
\hline \multirow{2}{*}{ Variable } & & \multirow[b]{2}{*}{ No. } & \multicolumn{3}{|c|}{ Univariate Analysis } \\
\hline & & & 5 Year-OS (\%) & $95 \%$ CI (\%) & $p$ Value \\
\hline \multirow{2}{*}{ Age } & $60>$ & 78 & 76.1 & $62.2-85.4$ & 0.196 \\
\hline & $60 \leq$ & 123 & 73.7 & $63.1-81.7$ & \\
\hline \multirow[t]{2}{*}{ Gender } & Male & 101 & 67.5 & $55.2-77.2$ & $<0.001$ \\
\hline & Female & 100 & 81.4 & $70.1-88.8$ & \\
\hline \multirow{2}{*}{$\begin{array}{l}\text { Metastasis at first } \\
\text { visit }\end{array}$} & Yes & 13 & 41.5 & $12-69.5$ & $<0.001$ \\
\hline & No & 188 & 76.8 & $68.5-83.1$ & \\
\hline \multirow[t]{2}{*}{ Tumor Grade } & 1 & 67 & 95.3 & $81-98.9$ & $<0.001$ \\
\hline & 2 and 3 & 124 & 62.4 & $51.5-71.5$ & \\
\hline \multirow[t]{2}{*}{ Histology } & WDLPS & 52 & 94.5 & $78.8-98.7$ & $<0.001$ \\
\hline & DDLPS, LMS and Others & 149 & 67.4 & $57.4-75.6$ & \\
\hline \multirow[t]{2}{*}{ Tumor size } & $<5 \mathrm{~cm}$ & 37 & 87.6 & $65.9-95.9$ & 0.0342 \\
\hline & $\geq 5 \mathrm{~cm}$ & 156 & 72.4 & $63.2-79.8$ & \\
\hline \multirow[t]{2}{*}{ Surgical resection } & Yes & 155 & 80.5 & $71.8-86.7$ & $<0.001$ \\
\hline & No & 46 & 52.4 & $32.2-69.1$ & \\
\hline \multirow[t]{2}{*}{ Chemotherapy } & Yes & 85 & 63.7 & $51.1-73.9$ & $<0.001$ \\
\hline & No & 116 & 85.6 & $75.6-91.7$ & \\
\hline \multirow[t]{2}{*}{ Radiotherapy } & Yes & 56 & 64.4 & $49-76.3$ & $<0.001$ \\
\hline & No & 145 & 79.9 & $70.4-86.7$ & \\
\hline \multirow[t]{2}{*}{ SUVmax } & Low (4>) & 72 & 92.9 & $82-97.3$ & $<0.001$ \\
\hline & $\operatorname{High}(4 \leq)$ & 73 & 58.4 & $44-70.3$ & \\
\hline
\end{tabular}


Table 4. Risk factor for OS in all RPS patients.

\begin{tabular}{ccc}
\hline Covariates & HR $(\mathbf{9 5} \%$ CI) & $p$ Value \\
\hline Age (60> vs. 60 $\leq)$ & $0.9331(0.4439-1.962)$ & 0.8551 \\
\hline Gender (Male vs. Female) & $0.6554(0.3642-1.789)$ & 0.5982 \\
\hline Metastasis at first visit (Yes vs. No) & $2.568(0.8083-8.162)$ & 0.1098 \\
\hline Tumor Grade (1 vs. 2 and 3) & $2.49(0.505-12.28)$ & 0.2624 \\
\hline Histology (WDLPS vs. DDLPS, LMS, and Other) & $10.33(0.5766-184.9)$ & 0.1127 \\
\hline Tumor size (<5 cm vs. $\geq 5$ cm) & $1.948(0.7235-5.243)$ & 0.187 \\
\hline Surgical resection (Yes or No) & $0.165(0.06428-0.4237)$ & $<0.001$ \\
\hline Chemotherapy (Yes or No) & $0.8073(0.3642-1.789)$ & 0.5982 \\
\hline Radiotherapy (Yes or No) & $1.009(0.459-2.218)$ & 0.9824 \\
\hline SUVmax (4> vs. $4 \leq)$ & $9.871(2.855-34.13)$ & $<0.001$ \\
\hline
\end{tabular}

It was suggested that SUVmax reflected aggressiveness and was useful for the prognostic implication of RPS. However, more patients with WDLPS or Grade1 tumors were recruited in the SUVmax low group than in the SUVmax high group (Table 2). Generally, tumor grade is a strong prognostic factor, and WDLPS is a low-grade malignant tumor. SUVmax in WDLPS (median, 1.9; range, 0-15.6) was lower than that in DDLPS (median, 5.7; range, 0-25.5), LMS (median, 6.75; range, 1.8-20.6), and other subtypes (median, 6.05; range 0-25.5) (Figure 3). Therefore, we investigated the correlation between SUVmax and RPS prognosis by histological subtype.

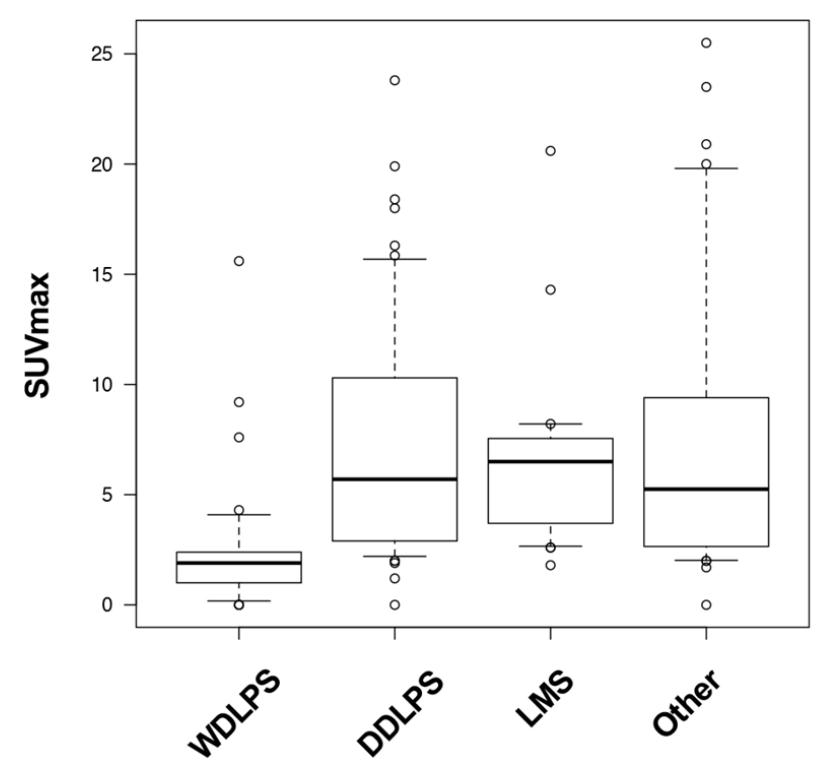

Figure 3. Distribution of SUVmax by histologic subtype.

We classified patients with RPS into four groups according to histological subtypes: WDLPS, DDLPS, LMS, and others. Regarding OS, univariate analysis revealed that high SUVmax was correlated with a shorter prognosis in the DDLPS and other groups (Figures $4 \mathrm{a}-\mathrm{c}$ and S1, Tables 5 and S1). No patient with WDLPS died during the observation period, and the prognosis of SUVmax high tended to be shorter on LMS but was not statistically significant (Figure $4 b, c$, Table 6). In addition, DFS was shorter only for patients with high SUVmax with LMS. Multivariate analyses indicated that SUVmax and surgical resection were independent prognostic factors in patients with DDLPS, but not those with other subtypes (Tables 7 and S2). 

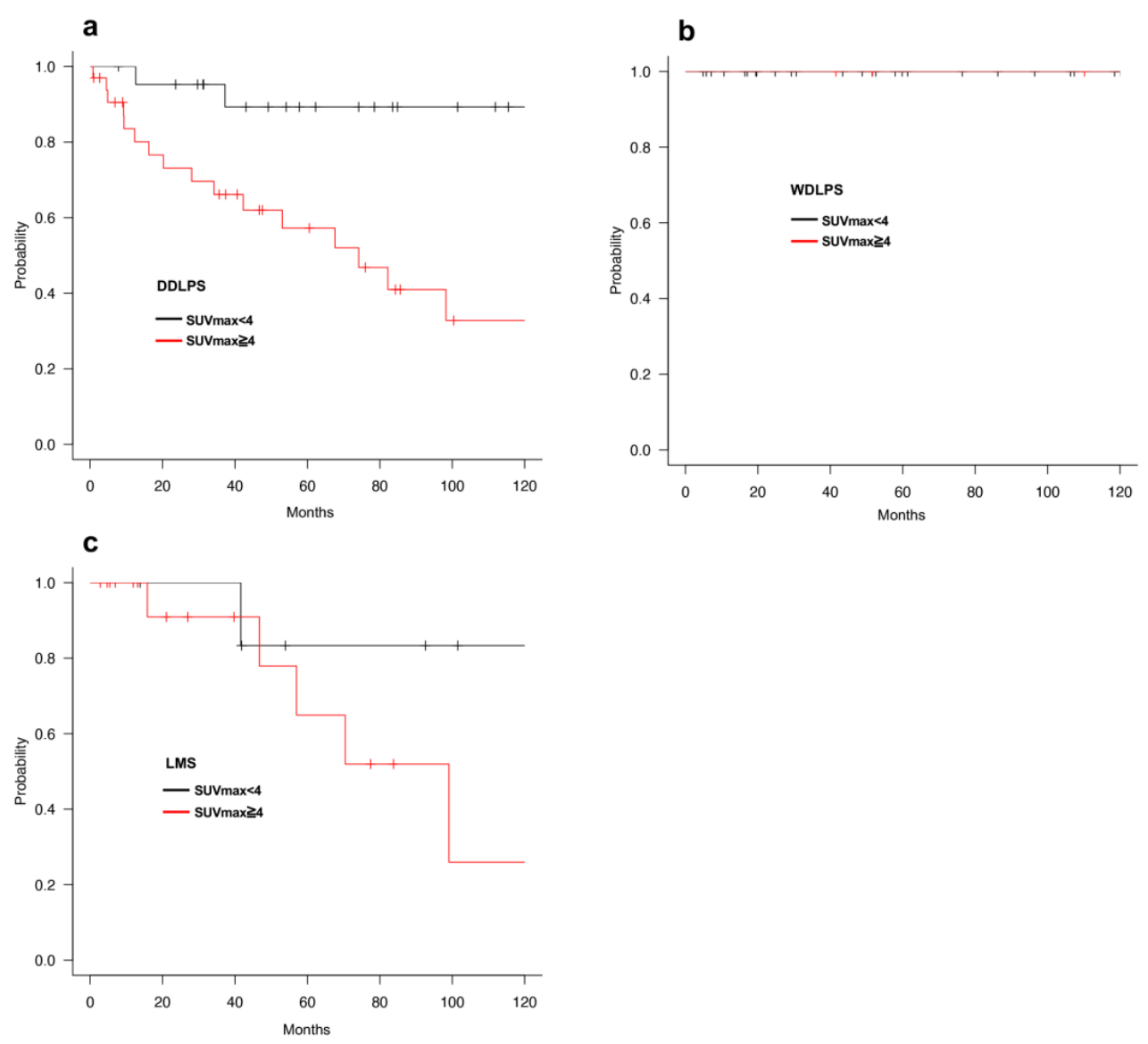

Figure 4. (a-c) OS of RPS patients with SUVmax low $(<4)$ or SUVmax high $(\geq 4)$ by histological subtypes, (a); DDLPS, (b); WDLPS and (c); LMS.

Table 5. Analyses on survival in all DDLPS patients.

\begin{tabular}{|c|c|c|c|c|c|}
\hline \multirow{2}{*}{ Variable } & & \multirow[b]{2}{*}{ No. } & \multicolumn{3}{|c|}{ Univariate Analysis } \\
\hline & & & 5 Year-OS (\%) & $95 \%$ CI (\%) & $p$ Value \\
\hline \multirow[t]{2}{*}{ Age } & $60>$ & 22 & 79.9 & $54.8-92$ & 0.176 \\
\hline & $60 \leq$ & 53 & 67.1 & $50.2-79.3$ & \\
\hline \multirow[t]{2}{*}{ Gender } & Male & 46 & 69.1 & $51.7-81.3$ & 0.131 \\
\hline & Female & 29 & 75.5 & $53-88.3$ & \\
\hline \multirow[t]{2}{*}{ Metastasis at first visit } & Yes & 2 & NA & NA & $<0.001$ \\
\hline & No & 73 & 71.3 & $58.1-81.6$ & \\
\hline \multirow[t]{2}{*}{ Tumor Grade } & 2 & 53 & 72.9 & $56.8-83.7$ & 0.219 \\
\hline & 3 & 19 & 60.8 & $31.7-80.6$ & \\
\hline \multirow[t]{2}{*}{ Tumor size } & $<5 \mathrm{~cm}$ & 11 & 83.3 & $27.3-97.5$ & 0.123 \\
\hline & $\geq 5 \mathrm{~cm}$ & 63 & 67.9 & $53.2-78.9$ & \\
\hline \multirow[t]{2}{*}{ Surgical resection } & Yes & 65 & 77.6 & $63.6-86.8$ & $<0.001$ \\
\hline & No & 10 & NA & NA & \\
\hline \multirow[t]{2}{*}{ Chemotherapy } & Yes & 37 & 58.9 & $39.5-74.0$ & 0.17 \\
\hline & No & 38 & 85.4 & $68.3-74$ & \\
\hline \multirow[t]{2}{*}{ Radiotherapy } & Yes & 28 & 59.2 & $37.1-75.8$ & 0.00346 \\
\hline & No & 47 & 79.9 & $63.5-89.5$ & \\
\hline \multirow[t]{2}{*}{ SUVmax } & Low $(4>)$ & 22 & 89.3 & $63.2-97.2$ & $<0.001$ \\
\hline & $\operatorname{High}(4 \leq)$ & 33 & 57.2 & $36.6-73.4$ & \\
\hline
\end{tabular}


Table 6. Analyses on survival in all LMS patients.

\begin{tabular}{cccccc}
\hline \multirow{2}{*}{ Variable } & & \multicolumn{4}{c}{ Univariate Analysis } \\
\cline { 2 - 6 } & & No. & 5 Year-OS (\%) & 95\% CI (\%) & $p$ Value \\
\hline Age & $60>$ & 18 & 49.9 & $16.5-76.5$ & 0.12 \\
& $60 \leq$ & 11 & 100 & NA & \\
\hline Gender & Male & 8 & 100 & NA & 0.795 \\
& Female & 21 & 61.2 & $28.8-82.5$ & \\
\hline Metastasis at first visit & Yes & 3 & 100 & NA & 0.441 \\
& No & 26 & 65.2 & $33.6-84.6$ & \\
\hline Tumor Grade & 2 & 19 & 72.3 & $34.3-90.7$ & 0.667 \\
& 3 & 8 & 41.7 & $1.1-84.3$ & \\
\hline Tumor size & $<5 \mathrm{~cm}$ & 5 & 66.7 & $5.4-94.5$ & 0.551 \\
& $\geq 5 \mathrm{~cm}$ & 21 & 72.6 & $32-91.4$ & \\
\hline Surgical resection & Yes & 24 & 67.6 & $37.2-99.1$ & 0.768 \\
& No & 5 & NA & NA & \\
\hline Chemotherapy & Yes & 15 & 41.8 & $10.2-71.6$ & 0.0231 \\
& No & 14 & 100 & NA & \\
\hline Radiotherapy & Yes & 7 & 50 & $5.8-84.5$ & 0.241 \\
& No & 22 & 73.1 & $34.7-91.1$ & \\
\hline SUVmax & Low $(4>)$ & 7 & 83.3 & $27.3-97.5$ & 0.244 \\
& High $(4 \leq)$ & 17 & 64.9 & $24.9-87.4$ & \\
\hline & & & &
\end{tabular}

Table 7. Multivariate Cox regression model of OS in DDLPS.

\begin{tabular}{ccc}
\hline \multirow{2}{*}{ Covariates } & \multicolumn{2}{c}{ Multivariate Analysis } \\
\cline { 2 - 3 } & HR (95\% CI) & $p$ Value \\
\hline Age $(60>$ vs. $60 \leq)$ & $2.022(0.6103-6.697)$ & 0.2494 \\
\hline Gender (Male vs. Female) & $0.6674(0.2305-1.932)$ & 0.4558 \\
\hline Metastasis at first visit (Yes vs. No) & $20.41(0.7559-551.2)$ & 0.07288 \\
\hline Tumor Grade (2 vs. 3) & $1.085(0.3348-3.516)$ & 0.8918 \\
\hline Tumor size $(<5$ cm vs. $\geq 5$ cm) & $8.129(0.7731-85.47)$ & 0.08088 \\
\hline Surgical resection (Yes or No) & $0.09174(0.02071-0.4064)$ & 0.001656 \\
\hline Chemotherapy (Yes or No) & $1.058(0.3098-3.614)$ & 0.9282 \\
\hline Radiotherapy (Yes or No) & $1.686(0.5881-4.832)$ & 0.3311 \\
\hline SUVmax $(4>$ vs. $4 \leq)$ & $6.056(1.257-29.19)$ & 0.0248 \\
\hline
\end{tabular}

We also compared the characteristics of the SUVmax low or SUVmax high groups in DDLPS. Most of the tumors were Grade2 in the SUVmax low group, but 45.5\% (15 out of 33) tumors were Grade3 with high SUVmax (Table 8). Since tumor Grade was a strong prognostic factor, we hypothesized that a short prognosis in patients with high SUVmax resulted from Grade3 tumors. On the contrary, the prognosis of patients with high SUVmax and Grade2 tumors were worse than those with low SUVmax and Grade2 tumors (Figure 5). In addition, there was no significant difference in the prognosis of patients with Grade2 and Grade3 tumors in the SUVmax high group (Figure 5). These results indicated that SUVmax had the potential clinical usefulness in better assessing the prognosis for RPS patients with DDLPS and Grade2 tumors. 
Table 8. Clinicopathologic and treatment characteristics between SUVmax low and SUVmax high in DDLPS.

\begin{tabular}{|c|c|c|c|}
\hline \multicolumn{4}{|c|}{ Dedifferentiated Liposarcoma } \\
\hline Characteristic & SUVmax Low $(n=22)$ & SUVmax High $(n=33)$ & $p$ Value \\
\hline Age, years $(60>/ 60 \leq)$ & $7 / 15$ & $11 / 22$ & 0.909 \\
\hline Gender (male/female) & $11 / 11$ & $24 / 9$ & 0.089 \\
\hline Localized/metastatic & $22 / 0$ & $31 / 2$ & 0.247 \\
\hline Tumor grade & & & 0.001 \\
\hline Grade1 & 0 & 0 & \\
\hline Grade2 & 21 & 18 & \\
\hline Grade3 & 1 & 15 & \\
\hline $\mathrm{NE}$ & 0 & 0 & \\
\hline Tumor size & & & 0.756 \\
\hline$<5 \mathrm{~cm}$ & 4 & 6 & \\
\hline$\geq 5 \mathrm{~cm}$ & 18 & 27 & \\
\hline NE & 0 & 0 & \\
\hline Surgical resection (Yes/No) & $21 / 1$ & $27 / 6$ & 0.142 \\
\hline Chemotherapy (Yes/No) & $7 / 15$ & $22 / 11$ & 0.011 \\
\hline Radiotherapy (Yes/No) & $4 / 18$ & $15 / 18$ & 0.038 \\
\hline
\end{tabular}

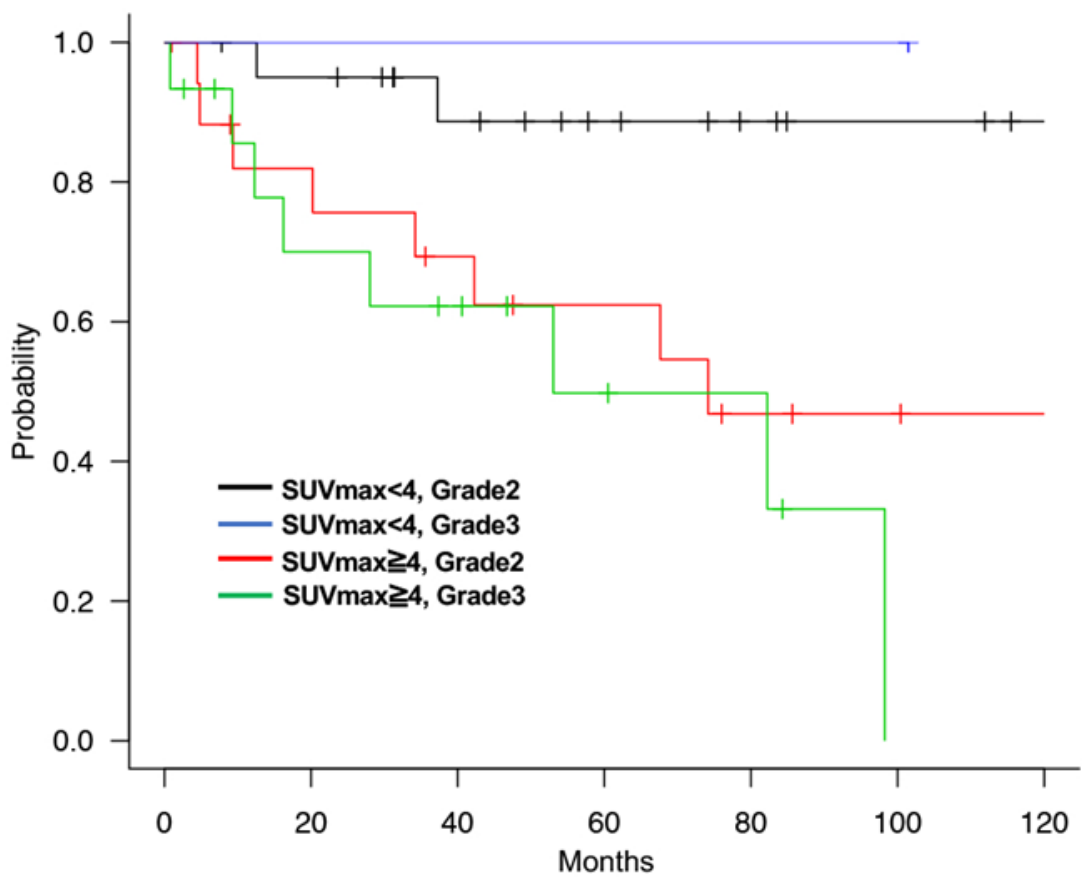

Figure 5. OS of DDLPS patients with SUVmax low and Grade2, SUVmax high and Grade2 and SUVmax and Grade3.

In conclusion, the current study demonstrated that SUVmax measured with FDG-PET was useful in determining the prognostic value of RPS, especially in DDLPS and other groups, but not in LMS. Since SUVmax with FDG-PET reflected the prognosis of patients with DDLPS and Grade2 RPS prior to FNCLCC Grade, FDG-PET may be considered an essential tool for clinical assessment in RPS.

\section{Discussion}

The present study demonstrated that a high SUVmax threshold $(\geq 4)$ was a strong prognostic factor for RPS, particularly DDLPS and Grade2 RPS tumors. Recently, Sambri et al. demonstrated that sarcoma patients with SUVmax of less than 10.3 showed a 
better prognosis and low recurrence rate in some histological types [16]. Subramaniam et al. also presented an association of higher SUVmax with higher histological Grade, worse recurrence-free survival, and poor OS in RPS, including DDLPS and LMS [17].

In the past, nomograms were created for primary and recurrent RPS to evaluate the patients' OS or DFS $[4,5,18,19]$. The nomogram aided in the prediction of OS or DFS probability corresponding to a combination of patient's covariates as follows: tumor size, FNCLCC Grade, histological subtype, multifocality, age, presentation (primary or recurrent), complete resection of surgery, chemotherapy or radiotherapy after the first surgery, and the number of resected organs at the first surgery. However, SUVmax with FDG-PET was not included in the nomogram. Our investigation demonstrated that SUVmax was correlated with the prognosis in Grade2 RPS and DDLPS. Brenner et al. reported SUVmax obtained by FDG-PET was a more useful parameter for risk assessment in liposarcoma than tumor Grade [20]. Therefore, SUVmax for Grade2 RPS and DDLPS may be considered an additional parameter within the nomogram, resulting in prognostic implications that could help decision-making regarding treatment intensity, such as combined resection of organs for complete resection of surgery, dose intensity, or regimens for chemotherapy or radiotherapy.

On the other hand, LMS was one of the most frequent malignant tumors in the retroperitoneum, and we could not suggest a clear prognostic difference between SUVmax low and SUVmax high in LMS patients. Since LMS showed high metastatic potential but not recurrence in our experience, many patients were lost to follow-up without treatment. There was a huge difference in the treatment strategy for each doctor in our institution, resulting in biased data on LMS.

In clinical practice, radical cure of RPS is difficult because the tumors are often too large to perform complete resection with wide margins, such as soft tissue sarcoma in the extremities. A previous study demonstrated histopathologic organ invasion in approximately $25 \%$ of adherent organs, even when not suspected intraoperatively [21]. Although combined resection of organs was needed to complete resection in many cases of RPS, it was reported that there was a higher operative risk in surgery involving pancreaticoduodenectomy, major vascular resection, and splenectomy/pancreatectomy, which might cause a potential treatment conflict [22]. Consequently, novel treatments or drugs that can shrink retroperitoneal tumors and prevent organ invasion are required for the radical cure of RPS.

Our data showed that RPS, at least DDLPS, with high SUVmax had a poor prognosis, indicating that cancer metabolism strongly links with tumor aggressiveness in RPS. Thus, targeting the metabolism of RPS might be promising for novel treatment strategies, and many studies have reported the anti-tumor effect of metabolic targeting in several sarcomas, including liposarcoma and Ewing sarcoma [23-26]. In most cases of DDLPS and WDLPS, gene amplification of MDM2 proto-oncogene (MDM2) and cyclin-dependent kinase 4 (CDK4) has been detected [27-30]. Based on our data, this might be a new strategy for seeking effective treatment targets from additional gene alterations in high SUVmax tumors than those with low SUVmax.

The present study has several limitations. First, this was a retrospective study using the clinical data of a single institute. Therefore, it is possible that unintentional bias in the selection of patients could not be fully eliminated. Second, the number of patients was too small to perform sufficient statistical analyses for several histological subtypes of RPS, such as LMS or undifferentiated pleomorphic sarcoma.

In conclusion, we demonstrated that FDG-PET imaging is useful in evaluating prognosis, and SUVmax reflects tumor aggressiveness in RPS, particularly DDLPS. We propose that FDG-PET may be considered before developing a treatment strategy for RPS.

\section{Conclusions}

The prognosis of retroperitoneal sarcoma patients with high maximum SUV (SUVmax) $(\geq 4)$ was worse compared to the patients with low SUVmax $(<4)$. Furthermore, 
among the patients with dedifferentiated liposarcoma or Grade2 retroperitoneal sarcoma, the overall survival rate for patients with high SUVmax $(\geq 4)$ was significantly lower than those with low SUVmax $(<4)$. These data demonstrated that SUVmax calculated with FDG-PET was useful as a prognostic factor in retroperitoneal sarcoma, especially in dedifferentiated liposarcoma and Grade2 retroperitoneal sarcoma. SUVmax on FDGPET scan may be considered for clinical assessment to develop a treatment strategy for retroperitoneal sarcoma.

Supplementary Materials: The following are available online at https: / www.mdpi.com/article / 10.3390 / cancers 13184611/s1, Figure S1: OS of RPS patients with SUVmax low $(<4)$ or SUVmax high $(\geq 4)$ in group of other, Table S1: Analyses of survival in other patients, Table S2: Multivariate Cox regression model of OS in other.

Author Contributions: T.W., Y.I., H.T. and S.T. designed the study. T.W. collected, interpreted, and analyzed the data. T.W. and S.T. performed the statistical analyses. T.W. wrote the manuscript. T.W., Y.I., H.T., T.Y., N.Y., S.N., T.N., H.O., K.H., S.K., N.A., T.U. and S.T. treated the patients. All authors have read and agreed to the published version of the manuscript.

Funding: The present study was supported by JSPS KAKENHI (grant No. JP20K18048).

Institutional Review Board Statement: The study was conducted according to the guidelines of the Declaration of Helsinki and approved by the Institutional Review Board of Osaka International Cancer Institute (protocol code; 1506059008-2, date of approval; 5 June 2015).

Informed Consent Statement: Informed consent was obtained from all subjects involved in the study.

Data Availability Statement: The data presented in this study are available on request from the corresponding author. The data are not publicly available because the Institutional Ethics Committee did not provide a specific authorization.

Acknowledgments: We thank all the participants who treated RPS patients in the current study at Osaka International Cancer Institute and Osaka University Orthopaedic Oncology Group.

Conflicts of Interest: All authors declare that they have no conflict of interest.

\section{References}

1. Mullinax, J.E.; Zager, J.S.; Gonzalez, R.J. Current diagnosis and management of retroperitoneal sarcoma. Cancer Control 2011, 18, 177-187. [CrossRef]

2. Lewis, J.J.; Leung, D.; Woodruff, J.M.; Brennan, M.F. Retroperitoneal soft-tissue sarcoma: Analysis of 500 patients treated and followed at a single institution. Ann. Surg. 1998, 228, 355-365. [CrossRef]

3. Kotilingam, D.; Lev, D.C.; Lazar, A.J.F.; Pollock, R.E. Staging Soft Tissue Sarcoma: Evolution and Change. CA. Cancer J. Clin. 2006, 56, 282-291. [CrossRef] [PubMed]

4. Raut, C.P.; Callegaro, D.; Miceli, R.; Barretta, F.; Rutkowski, P.; Blay, J.Y.; Lahat, G.; Strauss, D.C.; Gonzalez, R.; Ahuja, N.; et al. Predicting survival in patients undergoing resection for locally recurrent retroperitoneal sarcoma: A study and novel nomogram from TARPSWG. Clin. Cancer Res. 2019, 25, 2664-2671. [CrossRef] [PubMed]

5. Gronchi, A.; Miceli, R.; Shurell, E.; Eilber, F.C.; Eilber, F.R.; Anaya, D.A.; Kattan, M.W.; Honoré, C.; Lev, D.C.; Colombo, C.; et al. Outcome prediction in primary resected retroperitoneal soft tissue sarcoma: Histology-specific overall survival and disease-free survival nomograms built on major sarcoma center data sets. J. Clin. Oncol. 2013, 31, 1649-1655. [CrossRef]

6. MacNeill, A.J.; Miceli, R.; Strauss, D.C.; Bonvalot, S.; Hohenberger, P.; Van Coevorden, F.; Rutkowski, P.; Callegaro, D.; Hayes, A.J.; Honoré, C.; et al. Post-relapse outcomes after primary extended resection of retroperitoneal sarcoma: A report from the Trans-Atlantic RPS Working Group. Cancer 2017, 123, 1971-1978. [CrossRef]

7. Gronchi, A.; Miceli, R.; Colombo, C.; Stacchiotti, S.; Collini, P.; Mariani, L.; Sangalli, C.; Radaelli, S.; Sanfilippo, R.; Fiore, M.; et al. Frontline extended surgery is associated with improved survival in retroperitoneal low- to intermediate-grade soft tissue sarcomas. Ann. Oncol. 2012, 23, 1067-1073. [CrossRef]

8. Miura, J.T.; Charlson, J.; Gamblin, T.C.; Eastwood, D.; Banerjee, A.; Johnston, F.M.; Turaga, K.K. Impact of chemotherapy on survival in surgically resected retroperitoneal sarcoma. Eur. J. Surg. Oncol. 2015, 41, 1386-1392. [CrossRef]

9. Bonvalot, S.; Gronchi, A.; Le Péchoux, C.; Swallow, C.J.; Strauss, D.; Meeus, P.; van Coevorden, F.; Stoldt, S.; Stoeckle, E.; Rutkowski, P.; et al. Preoperative radiotherapy plus surgery versus surgery alone for patients with primary retroperitoneal sarcoma (EORTC-62092: STRASS): A multicentre, open-label, randomised, phase 3 trial. Lancet Oncol. 2020, 21, $1366-1377$. [CrossRef] 
10. Ioannidis, J.P.A.; Lau, J. 18F-FDG PET for the diagnosis and grading of soft-tissue sarcoma: A meta-analysis. J. Nucl. Med. 2003, $44,717-724$.

11. Kwee, T.C.; Basu, S.; Saboury, B.; Ambrosini, V.; Torigian, D.A.; Alavi, A. A new dimension of FDG-PET interpretation: Assessment of tumor biology. Eur. J. Nucl. Med. Mol. Imaging 2011, 38, 1158-1170. [CrossRef] [PubMed]

12. Schwarzbach, M.H.M.; Hinz, U.; Dimitrakopoulou-Strauss, A.; Willeke, F.; Cardona, S.; Mechtersheimer, G.; Lehnert, T.; Strauss, L.G.; Herfarth, C.; Büchler, M.W. Prognostic significance of preoperative [18-F] fluorodeoxyglucose (FDG) positron emission tomography (PET) imaging in patients with resectable soft tissue sarcomas. Ann. Surg. 2005, 241, 286-294. [CrossRef] [PubMed]

13. Chen, L.; Wu, X.; Ma, X.; Guo, L.; Zhu, C.; Li, Q. Prognostic value of 18F-FDG PET-CT-based functional parameters in patients with soft tissue sarcoma a meta-analysis. Medicine 2017, 96, e5913. [CrossRef] [PubMed]

14. Li, C.P.; Liu, D.N.; Zhou, N.N.; Tian, X.Y.; Wang, Z.; Liu, B.N.; Hao, C.Y. Prediction of Histologic Subtype and FNCLCC Grade by SUVmax Measured on 18F-FDG PET/CT in Patients with Retroperitoneal Liposarcoma. Contrast Media Mol. Imaging 2021, 2021, 7191363. [CrossRef]

15. Rhu, J.; Hyun, S.H.; Lee, K.H.; Jo, S.J.; Lee, K.W.; Park, J.B.; Kim, S.J. Maximum standardized uptake value on 18 Ffluorodeoxyglucose positron emission tomography/computed tomography improves outcome prediction in retroperitoneal liposarcoma. Sci. Rep. 2019, 9, 6605. [CrossRef]

16. Sambri, A.; Bianchi, G.; Longhi, A.; Righi, A.; Donati, D.M.; Nanni, C.; Fanti, S.; Errani, C. The role of 18F-FDG PET/CT in soft tissue sarcoma. Nucl. Med. Commun. 2019, 40, 626-631. [CrossRef]

17. Subramaniam, S.; Callahan, J.; Bressel, M.; Hofman, M.S.; Mitchell, C.; Hendry, S.; Vissers, F.L.; Van der Hiel, B.; Patel, D.; Van Houdt, W.J.; et al. The role of ${ }^{18} \mathrm{~F}-\mathrm{FDG}$ PET/CT in retroperitoneal sarcomas-A multicenter retrospective study. J. Surg. Oncol. 2021, 123, 1081-1087. [CrossRef] [PubMed]

18. Anaya, D.A.; Lahat, G.; Wang, X.; Xiao, L.; Pisters, P.W.; Cormier, J.N.; Hunt, K.K.; Feig, B.W.; Lev, D.C.; Pollock, R.E. Postoperative nomogram for survival of patients with retroperitoneal sarcoma treated with curative intent. Ann. Oncol. 2010, 21, 397-402. [CrossRef]

19. Ardoino, I.; Miceli, R.; Berselli, M.; Mariani, L.; Biganzoli, E.; Fiore, M.; Collini, P.; Stacchiotti, S.; Casali, P.G.; Gronchi, A. Histology-specific nomogram for primary retroperitoneal soft tissue sarcoma. Cancer 2010, 116, 2429-2436. [CrossRef]

20. Brenner, W.; Eary, J.F.; Hwang, W.; Vernon, C.; Conrad, E.U. Risk assessment in liposarcoma patients based on FDG PET imaging. Eur. J. Nucl. Med. Mol. Imaging 2006, 33, 1290-1295. [CrossRef]

21. Fairweather, M.; Wang, J.; Jo, V.Y.; Baldini, E.H.; Bertagnolli, M.M.; Raut, C.P. Surgical Management of Primary Retroperitoneal Sarcomas: Rationale for Selective Organ Resection. Ann. Surg. Oncol. 2018, 25, 98-106. [CrossRef]

22. Macneill, A.J.; Gronchi, A.; Miceli, R.; Bonvalot, S.; Swallow, C.J.; Hohenberger, P.; Van Coevorden, F.; Rutkowski, P.; Callegaro, D.; Hayes, A.J.; et al. Postoperative Morbidity after Radical Resection of Primary Retroperitoneal Sarcoma. Ann. Surg. 2018, 267, 959-964. [CrossRef]

23. Tanner, J.M.; Bensard, C.; Wei, P.; Krah, N.M.; Schell, J.C.; Gardiner, J.; Schiffman, J.; Lessnick, S.L.; Rutter, J. EWS/FLI is a master regulator of metabolic reprogramming in Ewing sarcoma. Mol. Cancer Res. 2017, 15, 1517-1530. [CrossRef]

24. Issaq, S.H.; Mendoza, A.; Fox, S.D.; Helman, L.J. Glutamine synthetase is necessary for sarcoma adaptation to glutamine deprivation and tumor growth. Oncogenesis 2019, 8, 20. [CrossRef]

25. Igarashi, K.; Kawaguchi, K.; Kiyuna, T.; Miyake, K.; Miyaki, M.; Yamamoto, N.; Hayashi, K.; Kimura, H.; Miwa, S.; Higuchi, T.; et al. Metabolic targeting with recombinant methioninase combined with palbociclib regresses a doxorubicin-resistant dedifferentiated liposarcoma. Biochem. Biophys. Res. Commun. 2018, 506, 912-917. [CrossRef]

26. Yu, P.Y.; Lopez, G.; Braggio, D.; Koller, D.; Bill, K.L.J.; Prudner, B.C.; Zewdu, A.; Chen, J.L.; Iwenofu, O.H.; Lev, D.; et al. miR-133a function in the pathogenesis of dedifferentiated liposarcoma. Cancer Cell Int. 2018, 18, 89. [CrossRef]

27. Ray-Coquard, I.; Blay, J.Y.; Italiano, A.; Le Cesne, A.; Penel, N.; Zhi, J.; Heil, F.; Rueger, R.; Graves, B.; Ding, M.; et al. Effect of the MDM2 antagonist RG7112 on the P53 pathway in patients with MDM2-amplified, well-differentiated or dedifferentiated liposarcoma: An exploratory proof-of-mechanism study. Lancet Oncol. 2012, 13, 1133-1140. [CrossRef]

28. Dickson, M.A.; Tap, W.D.; Keohan, M.L.; D’Angelo, S.P.; Gounder, M.M.; Antonescu, C.R.; Landa, J.; Qin, L.X.; Rathbone, D.D.; Condy, M.M.; et al. Phase II trial of the CDK4 inhibitor PD0332991 in patients with advanced CDK4-amplified well-differentiated or dedifferentiated liposarcoma. J. Clin. Oncol. 2013, 31, 2024-2028. [CrossRef]

29. Beird, H.C.; Wu, C.C.; Ingram, D.R.; Wang, W.L.; Alimohamed, A.; Gumbs, C.; Little, L.; Song, X.; Feig, B.W.; Roland, C.L.; et al. Genomic profiling of dedifferentiated liposarcoma compared to matched well-differentiated liposarcoma reveals higher genomic complexity and a common origin. Cold Spring Harb. Mol. Case Stud. 2018, 4, a002386. [CrossRef]

30. Hirata, M.; Asano, N.; Katayama, K.; Yoshida, A.; Tsuda, Y.; Sekimizu, M.; Mitani, S.; Kobayashi, E.; Komiyama, M.; Fujimoto, H.; et al. Integrated exome and RNA sequencing of dedifferentiated liposarcoma. Nat. Commun. 2019, 10, 5683. [CrossRef] 